\title{
Causa infrecuente de hipertensión arterial en lactantes: neuroblastoma congénito quístico suprarrenal. Caso clínico
}

\author{
DANIELA CARRILLO V. ${ }^{1}$, XIMENA IBARRA G. ${ }^{1}$, MÓNICA CUEVAS P. ${ }^{2}$, FLORENCIA DE BARBIERI M. ${ }^{3}$, \\ ROBERTO OYANEDEL Q. ${ }^{3}$, ALEJANDRO ZAVALA B. ${ }^{4}$, PAULINA BAQUEDANO D. ${ }^{5}$ \\ 1. Pediatra, Residente del Programa de Especialista en Nefrología Infantil, División de Pediatría, Pontificia Universidad \\ Católica de Chile. \\ 2. Nefrólogo Pediatra, Unidad de Nefrología, División de Pediatría, Pontificia Universidad Católica de Chile. \\ 3. Radiólogo, Unidad de Imagenología, Pontificia Universidad Católica de Chile. \\ 4. Cirujano Pediátrico, División de Cirugía, Pontificia Universidad Católica de Chile. \\ 5. Urólogo Pediátrico, Departamento de Urología, Pontificia Universidad Católica de Chile.
}

\begin{abstract}
A rare cause of hypertension in childhood. Congenital cystic adrenal neuroblastoma. Clinical case

The frequency of hypertension (HBP) in children has increased significantly over the past decade. The younger the patient the greater the likelihood of having secondary HBP. Thus, the main causes of hypertension in newborns are of renovascular or parenchymatous origin. Objective: To present the case of an infant with hypertension caused by a congenital cystic neuroblastoma (NB). Case History: Newborn with prenatal diagnosis of adrenal cyst, who evolved with significant hypertension unresponsive to medical therapy. Neuroblastoma was suspected on the basis of magnetic resonance imaging findings and resection of the lesion was able to resolve the hypertension and to confirm the diagnosis by anatomo-pathological study. Conclusion: Most cases of neonatal hypertension are of renal origin, with the 2 largest categories being renovascular and renal parenchymal diseases. NB is the most common neonatal malignancy. It usually presents as an abdominal mass of antenatal diagnosis, being the hypertension an unusual form of presentation.

(Key words: Neuroblastoma, hypertension, congenital, cystic).

Rev Chil Pediatr 2012; 83 (3): 262-268
\end{abstract}

\section{RESUMEN}

La frecuencia de hipertensión arterial (HTA) en niños ha aumentado significativamente en la última década. A menor edad del paciente mayor es la probabilidad de que la HTA sea secundaria. Así, las principales causas de HTA en recién nacidos son de origen renovascular o parenquimatoso. Objetivo: Presentar el caso de un lactante hipertenso por neuroblastoma (NB) congénito quístico. Caso Clínico: Recién nacido con diagnóstico prenatal de quiste suprarrenal, quien evolucionó con HTA por sobre el percentil 99 para edad, sexo y talla, 
sin respuesta a terapia farmacológica. La resonancia magnética permitió realizar el diagnóstico presuntivo de neuroblastoma congénito y la resección de la lesión permitió resolver la HTA y confirmar el diagnóstico. Conclusión: La HTA en recién nacidos generalmente se debe a causas secundarias. El NB es el tumor maligno neonatal más frecuente que se puede presentar como una masa abdominal de diagnóstico antenatal, siendo la HTA una forma infrecuente de presentación.

(Palabras clave: Neuroblastoma, hipertensión arterial, congénito, quiste).

Rev Chil Pediatr 2012; 83 (3): 262-268

\section{Introducción}

La frecuencia de hipertensión arterial (HTA) en niños ha aumentado significativamente en la última década ${ }^{1,2}$. En relación a la etiología, se considera que a menor edad del paciente y a mayor magnitud de la HTA mayor es la probabilidad de que se trate de una HTA secundaria. Así, las principales causas de HTA en recién nacidos son de origen renovascular o parenquimatoso ${ }^{3}$, por el contrario, en la etapa adolescente la causa más frecuente es la HTA esencial.

Presentamos el caso de un recién nacido con diagnóstico prenatal de quiste suprarrenal, quién evolucionó al segundo mes de vida con HTA por sobre el percentil 99 para edad, sexo y talla; que no se pudo controlar con terapia farmacológica. La resección de la lesión permitió realizar el diagnóstico y resolver la HTA. El diagnóstico final fue neuroblastoma congénito, tumor sólido extracraneal más común en lactantes menores, generalmente asintomático presentándose en la mayoría de los casos como una masa abdominal palpable e infrecuentemente como HTA aislada.

El objetivo de esta publicación es dar a conocer a la HTA como una forma de presentación infrecuente de neuroblastoma congénito y enfatizar la relevancia que tiene la búsqueda etiológica de la HTA en recién nacidos y lactantes.

\section{Caso clínico}

Lactante menor con antecedente de ser el segundo hijo de padres no consanguíneos y sanos. Madre cursó un embarazo fisiológico controlado desde las seis semanas de edad gestacional. En la ecotomografía prenatal realiza- da a las $36^{+5}$ semanas se detectó una imagen anecogénica de $14 \mathrm{~mm}$ de diámetro mayor, localizada en el polo superior del riñón derecho; con líquido amniótico de volumen normal, sin otras alteraciones anatómicas. Nació por parto vaginal espontáneo a las $40^{+2}$ semanas y fue un recién nacido de término adecuado para la edad gestacional sin masa abdominal palpable al examen físico. Por la presencia de la lesión quística descrita se mantuvo en control clínico y seguimiento ecográfico. En ecotomografías seriadas, la lesión quística impresionó ser extrarrenal y presentó un aumento de tamaño hasta $24 \mathrm{~mm}$ de diámetro mayor (figura 1). Por la dificultad diagnóstica se realizó estudio con una resonancia magnética (RM) que demostró una lesión redondeada marcadamente hiperintensa en T2 en la región de la glándula suprarrenal derecha (figura 2). Esta lesión presentaba una fina pared regular que se realzaba con el uso de gadolinio y se observó un plano de clivaje con el polo superior del riñón derecho, no logrando visualizarse la glándula suprarrenal ipsilateral (figura 3). Debido a que el paciente evolucionó significativamente hipertenso (PA hasta $124 / 46$, lo que está por sobre el percentil 99 para sexo, edad y talla del paciente) se solicitó ecotomografía renal doppler la que no demostró estenosis hemodinámicamente significativa de las arterias renales ${ }^{4}$. Se inició tratamiento con captopril $(0,42 \mathrm{mg} / \mathrm{kg} /$ día $)$, disminuyendo cifras tensionales, pero sin lograr su normalización. Dada la fuerte sospecha clínica de neuroblastoma congénito sintomático se decidió extirpar la lesión con el fin de realizar estudio anátomo-patológico. A los dos meses de vida se efectuó suprarrenalectomía derecha laparoscópica. El estudio anátomo-patológico confirmó la presencia de un neuroblastoma poco diferenciado de la glándula suprarrenal derecha con bordes quirúrgicos libres de tu- 


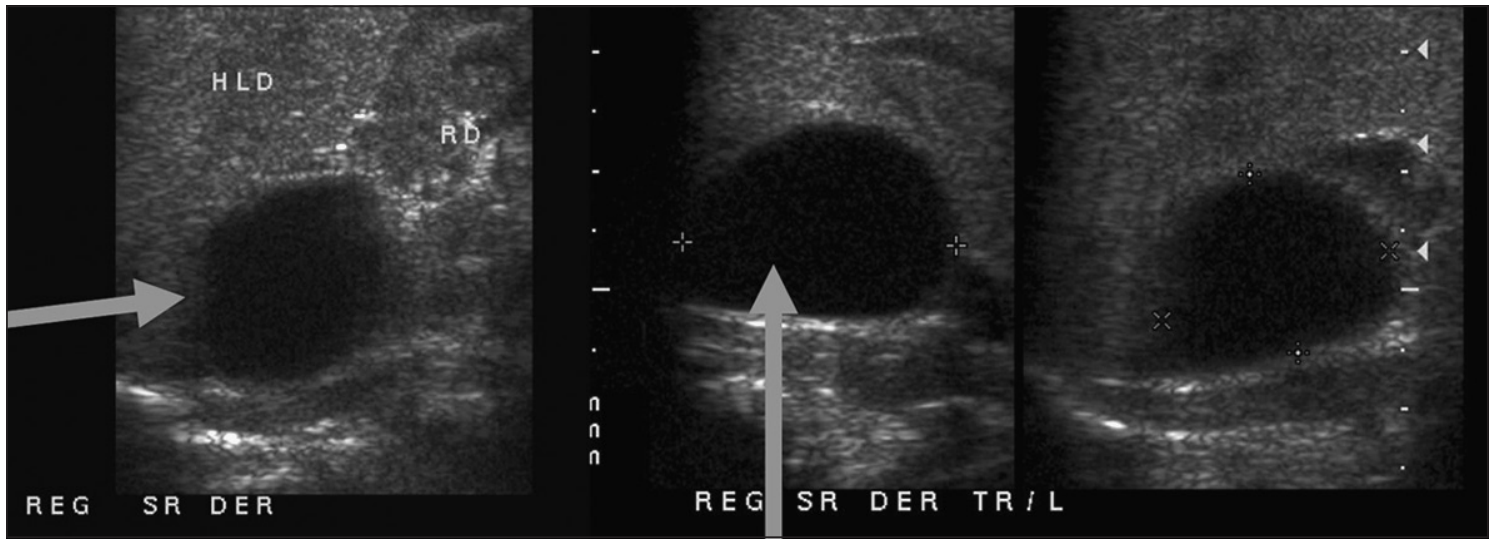

Figura 1. Ecotomografía abdominal: En la proximidad del polo superior de riñón derecho se identifica una imagen quística de bordes bien definidos y paredes relativamente gruesas (1), que mide 2,4 cm de diámetro mayor e impresiona ser extrarrenal (RD: riñón derecho. HLD hígado lóbulo derecho).
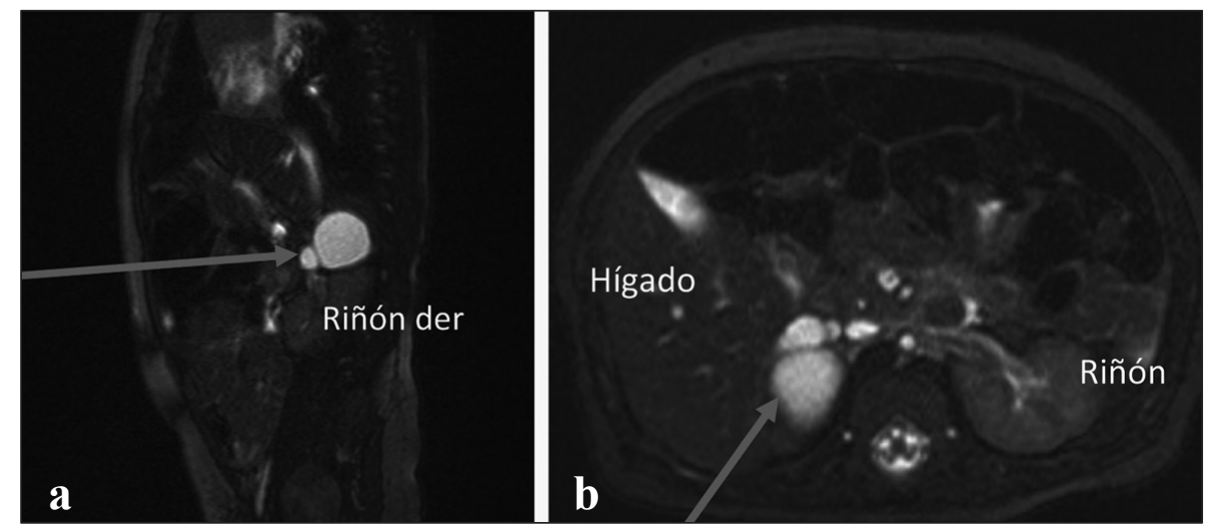

Figura 2. Resonancia magnética: Cortes de $\mathrm{RM}$ ponderadas en $\mathrm{T} 2$ en corte sagital (a) y axial (b). Se identifica una lesión quística biloculada en la región suprarrenal derecha.
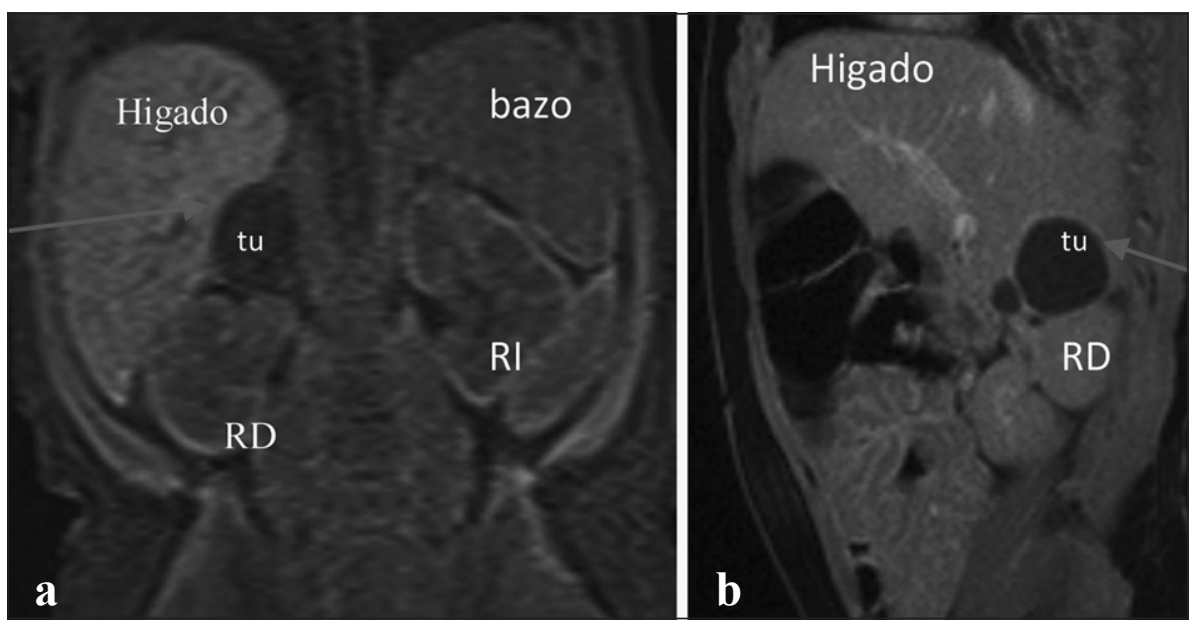

Figura 3. Resonancia magnética: Cortes ponderados en $\mathrm{T} 1$ pre (a) y post administración de gadolinio (b) demuestran discreto refuerzo parietal (Flecha), sin modificación del contenido del tumor (tu). 
mor, estadio 1 del Sistema Internacional de Estadificación de Neuroblastoma. El estudio cintigráfico óseo resultó normal y la amplificación del gen N-myc (por reacción de polimerasa en cadena) fue negativa, por lo que no requirió tratamiento coadyuvante. El paciente evolucionó normotenso desde el primer día post-operatorio y se ha mantenido en control clínico e imagenológico, con ecografía abdominal seriada, hasta los 19 meses de vida.

\section{Discusión}

Los tumores abdominales se presentan en forma infrecuente durante la infancia. El Neuroblastoma (NB) es el tumor maligno más frecuente en niños menores de un año, cuya incidencia es de aproximadamente 58/1 000000 niños por año $0^{5}$ En la casuística del grupo cooperativo chileno Programa Infantil de Drogas Antineoplásicas (PINDA) el neuroblastoma representa un 4,2\% de las neoplasias de la infancia ${ }^{6}$. El neuroblastoma perinatal o congénito, corresponde a $1 / 5$ de todos los casos de esta enfermedad; siendo el $41 \%$ diagnosticado en los primeros 3 meses de vida ${ }^{7}$. Cabe destacar que con el uso sistemático de la ecografía obstétrica se ha observado un incremento en el diagnóstico antenatal de este tumor ${ }^{8,9}$.

Basado en la edad de presentación, el NB congénito se clasifica en fetal y neonatal. Ambos son generalmente de buen pronóstico, pero difieren en el patrón de metástasis y en el órgano de origen. El NB fetal es diagnosticado por ultrasonido a una edad gestacional promedio de 36 semanas y es en el $90 \%$ de los casos de origen suprarrenal. Se asocia a metástasis hepática y placentaria. Mientras que el NB neonatal es en un $45 \%$ de origen suprarrenal y un $60 \%$ presenta metástasis al momento del diagnóstico ${ }^{8}$.

El NB es un tumor que se origina en el sistema nervioso simpático, está formado por células indiferenciadas, que se desarrollan a partir de las células embrionarias que migran de la cresta neural hasta formar ganglios simpáticos y médula suprarrenal. Su etiología es desconocida, pero la hipótesis más aceptada es que se trataría de la persistencia de nódulos de neuro- blastos presentes en condiciones normales en la glándula adrenal fetal entre las semanas 17 y 20 de la gestación ${ }^{6,10}$. Los sitios más comunes de presentación son: glándula suprarrenal (35\%), retroperitoneo $(30-35 \%)$ y mediastino posterior $(20 \%)^{8}$.

La mayoría de los pacientes son asintomáticos y se diagnostican por hallazgo en una ecotomografía de rutina. Durante el período prenatal se puede presentar como óbito fetal por compresión del cordón umbilical, hidrops fetal por compromiso placentario o por preeclampsia materna causada por exceso de catecolaminas ${ }^{6}$.

El hallazgo clínico de una masa abdominal palpable en el período neonatal inmediato tiene una probabilidad de un $80 \%$ de corresponder a un neuroblastoma. Otras formas de presentación durante este período incluyen: distress respiratorio severo, anemia secundaria a infiltración de médula ósea, nódulos en piel y en baja frecuencia hipertensión arterial por el aumento de las catecolaminas circulantes ${ }^{6}$.

El hallazgo ecográfico más frecuente $(50 \%)$ corresponde al de una masa sólida ecogénica de 2 a $4 \mathrm{~cm}$ de diámetro. El otro $50 \%$ de los casos se presenta como una lesión quística compleja, excepcionalmente como una lesión quística simple y más frecuentemente como una masa sólido-quística.

En la actualidad la sospecha diagnóstica de NB congénito se realiza por ecografía obstétrica de rutina durante el tercer trimestre del embarazo, se confirma con ecografías perinatales y el diagnóstico se basa en los hallazgos en RM, la que permite evaluar la localización exacta de la lesión, el compromiso de órganos vecinos, extensión intrarraquídea y compromiso óseo. El estudio de diseminación se complementa con tomografia computada tóracoabdominal ${ }^{11}$. En relación al uso de PET-CT, actualmente su utilidad se limita a tumores en que se sospecha el diagnóstico de NB y que no muestran captación en el cintigrama ${ }^{123} \mathrm{I}-$ MIBG, y al seguimiento de éstos ${ }^{12,13}$.

Si bien es cierto que la medición de catecolaminas urinarias (ácido vanilmandélico y/o ácido homovalínico) es de utilidad en el diagnóstico de esta patología, es importante recordar que en período neonatal éstas se en- 
cuentran elevadas sólo en un 50\% de los casos y que este porcentaje se reduce a un $9,5 \%$ en las formas quísticas, motivo por el cual en el paciente descrito no fueron medidas, ya que su medición requería realizar recolección de orina con sonda durante $24 \mathrm{~h}$ con el consiguiente riesgo de infección del tracto urinario y no modificarían la conducta terapéutica a seguir ${ }^{10}$.

Lo mismo sucede con el uso del cintigrama con ${ }^{123}$ I-MIBG, el que es útil en la identificación de la masa tumoral y en la búsqueda de metástasis con un valor predictivo positivo mayor al 90\%; sin embargo, para el neuroblastoma congénito la sensibilidad de este examen es tan sólo del 70\% y el valor predictivo negativo de $55 \%{ }^{14}$.

Pacientes con neuroblastoma congénito deben ser evaluados en búsqueda de enfermedad metastásica, para esto se recomienda realizar una RM y/o tomografía computada multicorte tóraco-abdominal para detectar metástasis hepáticas y estudiar el eventual compromiso tumoral de los ganglios linfáticos. El compromiso de la médula ósea en niños mayores se realiza mediante aspiración medular y biopsia de médula ósea, sin embargo, considerando la dificultad técnica, se han sugerido procedimientos diagnósticos alternativos en lactantes y recién nacidos. Así, el uso de cintigramas ${ }^{123} \mathrm{I}-\mathrm{MIBG}$ y ${ }^{99 \mathrm{~m}}$ TC-MDP puede ser útil en identificar enfermedad metastásica asociada.

Se han descrito factores genético moleculares que determinan un pronóstico menos favorable, entre ellos se pueden mencionar: amplificación del gen $\mathrm{N}-m y c$, deleción del cromosoma 1p, DNA euploide, falta de expresión de CD44 y N-ras ${ }^{15,16}$. Estos factores de riesgo rara vez se presentan en menores de 1 año, pero están presentes en un tercio de neuroblastomas etapas 3 y 4 de niños mayores de un año?.

El principal diagnóstico diferencial de las formas quísticas de presentación es la hemorragia de la glándula suprarrenal, la que usualmente se presenta en recién nacidos con antecedente de trauma obstétrico, asfixia perinatal, septicemia y/o trastornos de la coagulación ${ }^{17}$. La hemorragia suprarrenal generalmente se presenta al nacer o en los primeros días de vida, siendo poco frecuente en el feto. Desde el punto de vista ecográfico se caracteriza por dis- minución progresiva del tamaño de la lesión, con degradación de los productos hemáticos hasta llegar a estado quístico con reabsorción de coágulos y aparición de calcificaciones intralesionales en el seguimiento ecográfico ${ }^{7}$. Es importante tener presente que una masa sólida ecogénica o hipoecogénica detectada después de las 32 semanas de gestación tiene un 95\% de probabilidades de corresponder a un neuroblastoma ${ }^{18}$. Otros diagnósticos diferenciales a considerar incluyen: hidronefrosis, secuestro pulmonar extralobar subdiafragmático, tumores renales (tumor de Wilms, nefroma mesoblástico) y displasia renal focal.

Al igual que en niños mayores, los neuroblastomas congénitos se clasifican de acuerdo al sistema de estadificación internacional para neuroblastoma (INSS). Los factores que considera el INSS incluyen: extensión local del tumor, compromiso ganglionar, extensión de la resección del tumor primario y presencia de enfermedad metastásica a distancia. A diferencia de lo que sucede con niños mayores, la mayoría de los pacientes con NB congénito presentan tumores localizados y resecables, lo que explica, en parte, el mejor pronóstico de este grupo de pacientes. Adicionalmente, la mayoría de los pacientes con NB congénito presentan marcadores biológicos favorables, así más de un $90 \%$ de los tumores tienen un índice de DNA mayor a 1; menos del 5\% de los casos presentan amplificación del oncogen $\mathrm{N}-m y c$ y aproximadamente un $95 \%$ de los casos congénitos se caracterizan histológicamente por ser indiferenciados, con poco estroma, y por presentar un bajo índice mitosis-cariorrexis, todas características asociadas a un pronóstico favorable ${ }^{6,19}$.

En relación al tratamiento, la sospecha antenatal de neuroblastoma implica la derivación del paciente a un centro de alta complejidad donde pueda ser evaluado por un equipo multidisciplinario que incluya obstetra, neonatolólogo, cirujano infantil, nefrólogo, radiólogo y oncólogo infantil ${ }^{6}$. Los protocolos de tratamiento consideran la etapificación de acuerdo al INSS y al comportamiento de los marcadores biológicos conocidos. En la mayoría de los casos, los neonatos con tumores primarios localizados son sometidos a biopsia excisio- 
nal, la que permite realizar confirmación del diagnóstico, evaluar marcadores biológicos y efectuar un tratamiento definitivo de la lesión $^{20}$. Pacientes con lesiones de riesgo intermedio tienen un muy buen pronóstico, con una sobrevida de aproximadamente $90 \%$ luego de un curso de quimioterapia ${ }^{8}$.

El excelente pronóstico de lactantes con enfermedad localizada y resecable, asociado a la observación de que algunos neuroblastomas diagnosticados antenatal regresan espontáneamente $^{10}$, ha llevado a algunos clínicos a considerar apropiada la conducta expectante en un subgrupo de estos pacientes. Así, la observación con evaluación clínica y ultrasonografía mensual durante los primeros 6 meses de vida puede evitar la realización de cirugías en pacientes que presentan lesiones que podrían desaparecer de forma espontánea. Esto requiere una evaluación inicial que incluya ecotomografía, medición de catecolaminas urinarias, cintigrama ${ }^{123}$ I-MIBG y monitoreo clínico y ultrasonográfico seriado. Esta estrategia es recomendada para tumores pequeños $(<30 \mathrm{~mm})$ y en especial para lesiones quísticas, las cuales se ha visto que regresan con mayor frecuencia en comparación con las formas sólidas ${ }^{18}$. Así, se ha propuesto que se considere la resección quirúrgica en casos de crecimiento de la lesión o si ésta persiste después de los 6 meses de vida. La cirugía se recomienda en etapas iniciales si la lesión es $>30 \mathrm{~mm}^{14}$.

En relación a la técnica quirúrgica, en los últimos años existe una tendencia a privilegiar la resección laparoscópica en tumores pequeños y siempre considerando la experiencia previa del cirujano y del centro donde se efectuará la cirugía ${ }^{21}$.

\section{Conclusión}

En el caso descrito, la aparición de HTA severa determinó la realización de resección laparoscópica del tumor, lo que permitió resolver la HTA y confirmar el diagnóstico. Considerando que no presentaba enfermedad metastásica ni marcadores biológicos que pudieran predecir un curso desfavorable, no se realizó ningún tratamiento adicional y se ha manteni- do con seguimiento ecográfico y clínico seriado, evolucionando hasta este momento normotenso, en excelente condiciones clínicas y sin nuevas lesiones detectables en ecotomografías seriadas.

Debido al uso rutinario y a un mayor acceso al seguimiento ecográfico prenatal, es posible esperar un aumento en la frecuencia del diagnóstico de lesiones suprarrenales. En este contexto, es importante recordar que la detección de masas suprarrenales durante el tercer trimestre de la gestación debe hacer sospechar un NB congénito. El control clínico y ecográfico seriado durante el período postnatal es de vital importancia para poder realizar un diagnóstico y tratamiento oportunos. Si bien la mayoría de los neuroblastomas se manifiestan como masa abdominal palpable, no se debe olvidar que la HTA puede ser el único signo clínico presente en estos pacientes, por lo que es importante recordar el control de presión arterial en todo $\mathrm{RN}$.

\section{Referencias}

1.- Muntner P, He J, Wildman RP, Whelton PK: Trends in blood pressure among children and adolescents. JAMA 2004; 291: 2107-13.

2.- Hansen TW, Staessen JA, Zhang H, et al: Cardiovascular outcome in relation to progression to hypertension in the Copenhagen MONICA cohort. Am J Hypertens 2007; 20 (5): 483-91.

3.- Dionne J, Abitbol C, Flynn JT: Hypertension in infancy: diagnosis, management and outcome. Pediatr Nephrol 2012; 27 (1): 17-32. Epub 2011 Jan 22.

4.- National high blood pressure education program working group on high blood pressure in children and adolescents: The Fourth report on the diagnosis, evaluation and treatment of high blood pressure in children and adolescents. Pediatrics 2004; 114: 555-76.

5.- Gurney JG, Ross JA, Wall DA, Bleyer WA, Severson RK, Robison LL: Infant cancer in the US: histology-specific incidence and trends, 1973 to 1992 . J Pediatr Hematol Oncol 1997; 19: 428-32.

6.- Rostión C, Jáuregui L, Broussain V, Gac K, Paulos A: Neuroblastoma: forma de presentación y probabilidad de resección quirúrgica. Rev Ped Elec 2005; 2 (2): 1620.

7.- Nuchtern JG: Perinatal neuroblastoma. Seminars in pediatric surgery $2006 ; 15: 10-6$. 
8.- López P, Torres C, Elizagaray E, Astigarraga I, Navajas A, Fernández-Teijeiro A: Neuroblastóma quístico prenatal. An Esp Pediatr 2002; 56: 265-7.

9.- Olaya N, Gil M: Neuroblastoma congénito metastásico. Reporte de caso. Rev Colom Obstet Ginecol 2007; 58 (4): 328-32.

10.- Ferrer J, Ortega J, Iriondo M, Moreno J, Cruz O, Manzanares $R$ : Neuroblastoma quístico fetal: a propósito de dos casos. An Esp Pediatr 1998; 49: 299-301.

11.- Brame M, Masel J, Homsy Y: Antenatal detection and management of suprarenal masses. Urology 1999; 54 (6): 1097viii-1097x.

12.- Jofré M, Massardo T, Canessa J, et al: Utilidad de la tomografía de emisión de positrones en oncología pediátrica. Rev Chil Pediatr 2007; 78 (3): 301-10.

13.- Mullassery D, Dominici C, Jesudason EC, McDowell $H P$, Losty PD: Neuroblastoma: contemporary management. Arch Dis Child Educ Pract Ed 2009; 94: 177-85.

14.- Sauvat F, Sarnacki S, Brisse H, et al: Outcome of suprarenal localized masses diagnosed during the perinatal period. Cancer 2002; 94 (9): 2474-80.

15.- Brossard J, Bernstein M, Lemieux B: Neuroblastoma: an enigmatic disease. British medicall bulletin 1996; 52 (4): 787-801.

16.- Kozakewich HP, Pérez-Atayde AR, Donovan MJ, et al: Cystic Neuroblastoma: emphasis on gene expression, morphology and pathogenesis. Pediatr Dev Pathol 1998; 1(1): 17-28.

17.- Yamagiwa I, Obata K, Saito H: Prenatally detected cystic neuroblastoma. Peditr Surg Int 1998; 13: 215-7.

18.- Acharya S, Jayabose S, Kogan SJ, et al: Prenatally diagnosed neuroblastoma. Cancer 1997; 80: 304-10.

19.- Diagnóstico y tratamiento del neuroblastoma en el paciente pediátrico; México, Secretaría de salud 2010.; www.cenetec.salud.gob.mx/interior/gpc.html.

20.- Luis AL, Martínez L, Hernández F, et al: Neuroblastomas congénitos. Cir Pediatr 2004; 17: 89-92.

21.- Kadamba P, Habib Z, Rossi L: Experience with laparoscopic adrenalectomy in children. Journal of Pediatric Surgery 2004; 39 (5): 764-67. 